\title{
Postpartum Congestive Heart Failure Due to Diastolic Dysfunction: Importance of Advanced Echo-Doppler Analysis
}

\author{
Matthew Gottbrecht ${ }^{1}$, Leslie Panella ${ }^{2}$, Laura Ferraro ${ }^{2}$, Colleen Harrington ${ }^{2}$, Gerard \\ Aurigemma $^{3}$, and Lara Kovell ${ }^{2}$ \\ ${ }^{1}$ UMass Memorial Medical Center \\ ${ }^{2}$ UMass Medical School \\ ${ }^{3}$ U. Mass Medical School
}

April 17, 2021

\begin{abstract}
We present a case of a 25 year-old female who developed acute hypoxic respiratory failure secondary to postpartum heart failure with preserved ejection fraction. Despite essentially normal tissue Doppler velocities and E/e' ratio by existing guidelines for the adult population, available literature suggests that our patient's markers of diastolic function were significantly abnormal relative to what is expected in the third trimester of pregnancy. Advanced echo-Doppler analysis and her clinical presentation support the presence of underlying diastolic dysfunction. This case illustrates the challenges associated with the diagnosis of diastolic dysfunction during pregnancy and the need for pregnancy specific guidelines.
\end{abstract}

Postpartum congestive heart failure due to diastolic dysfunction: Importance of advanced echo-Doppler analysis

Matthew Gottbrecht, $\mathrm{MD}^{1}$, Leslie Panella, $\mathrm{BS}^{2}$, Laura Ferraro, $\mathrm{MD}^{3}$, Colleen M Harrington, $\mathrm{MD}^{1}$, Gerard $\mathrm{P}$ Aurigemma, $\mathrm{MD}^{1}$, Lara C Kovell, $\mathrm{MD}^{1}$

1. Division of Cardiovascular Medicine, Department of Medicine, University of Massachusetts Medical School, Worcester, Massachusetts.

2. University of Massachusetts Medical School, Worcester, Massachusetts.

3. Division of General Obstetrics and Gynecology, Department of Obstetrics and Gynecology, University of Massachusetts Medical School, Worcester, Massachusetts.

Running Title: Postpartum HFpEF: value of advanced Doppler analysis

Total Word Count: 1,123 words

Funding: None

Disclosures: No relevant disclosures or competing interests to report.

\section{Address for Correspondence:}

Matthew Gottbrecht, MD

Cardiology Fellow

Division of Cardiovascular Medicine

University of Massachusetts Medical School 
Email: matthew.gottbrecht@umassmemorial.org

Phone: (781) 254-2803

Fax: (508) 856-4571

Acknowledgements: None

Keywords:

Pregnancy

Diastolic heart failure

Doppler echocardiography

Strain imaging

Cardiovascular disease in women

Abbreviations List:

BNP: Brain natriuretic peptide

DD: Diastolic dysfunction

E: Early diastolic mitral inflow velocity

e': early diastolic mitral annular tissue velocity

EDPVR: End diastolic pressure volume relationship

EF: Ejection fraction

GLS: Global longitudinal strain

HFpEF: Heart failure with preserved ejection fraction

pHFpEF: Postpartum heart failure with preserved ejection fraction

LA: Left atrial

LAVi: Left atrium end-systolic volume index

LV: Left ventricle

PASP: Pulmonary artery systolic pressure

SpO2: Oxygen saturation

Hosted file

Echocardiography_case report_3_30_sub.pdf available at https://authorea.com/users/408422/

articles/518441-postpartum-congestive-heart-failure-due-to-diastolic-dysfunction-

importance-of-advanced-echo-doppler-analysis 

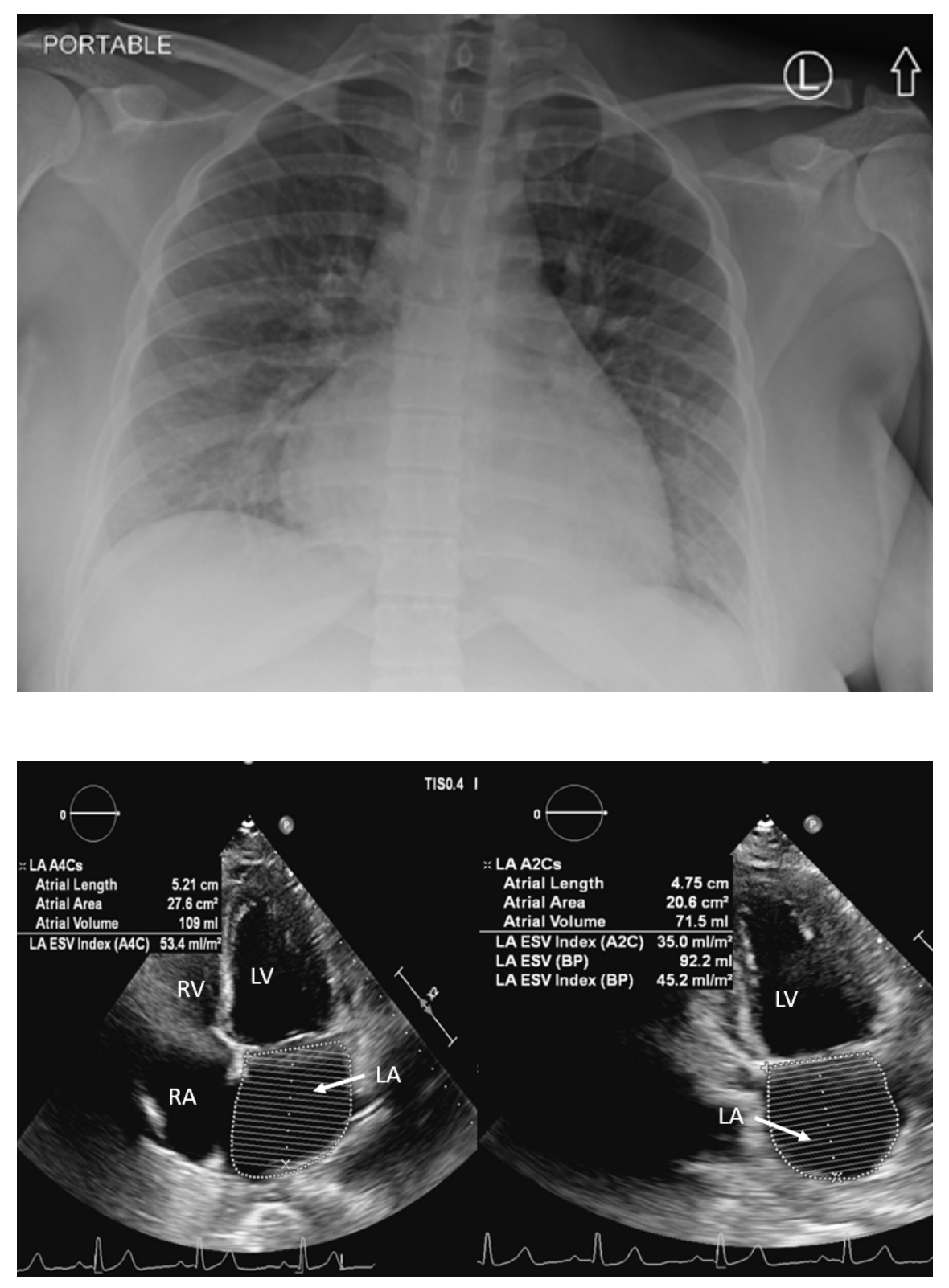

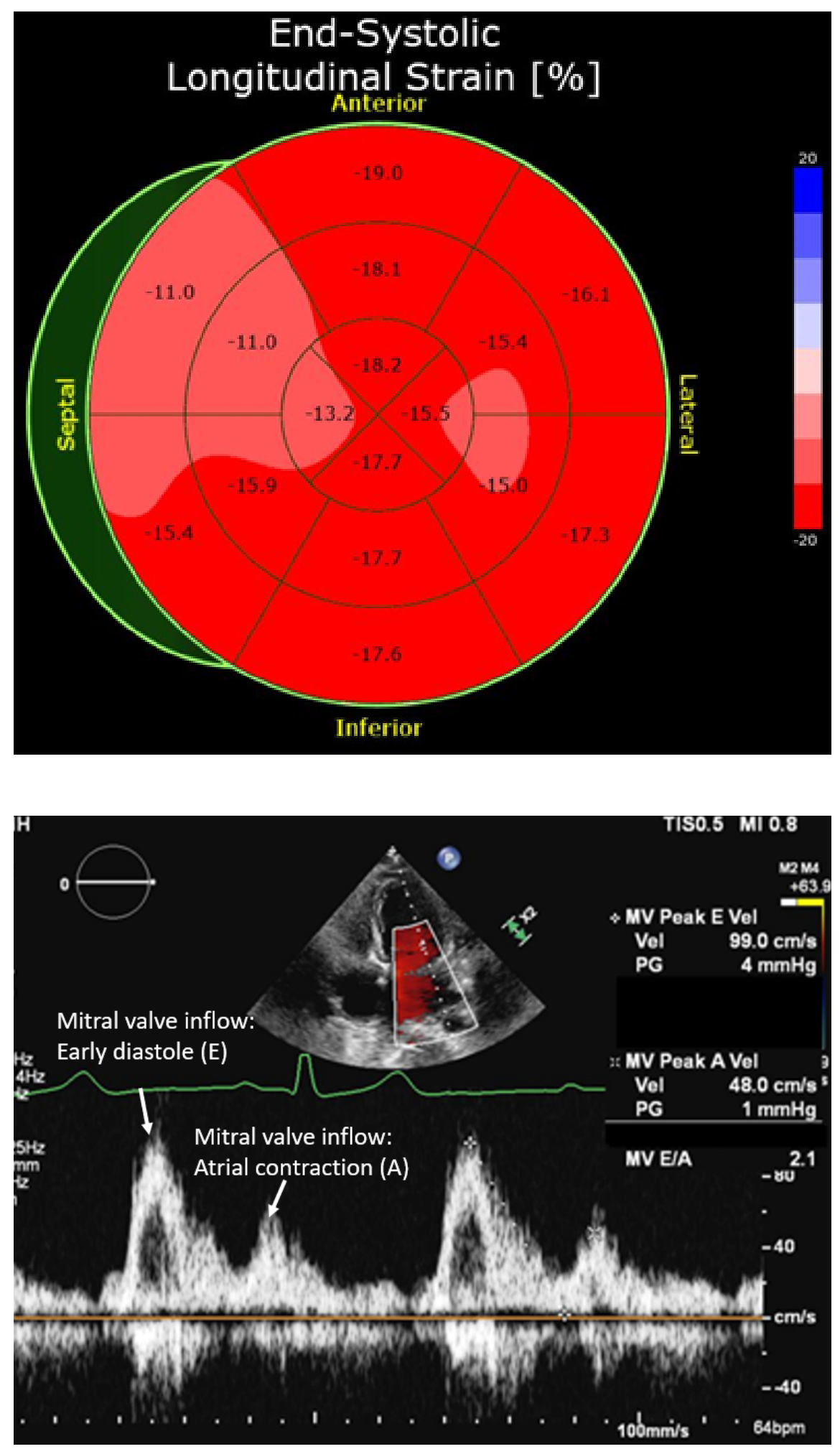


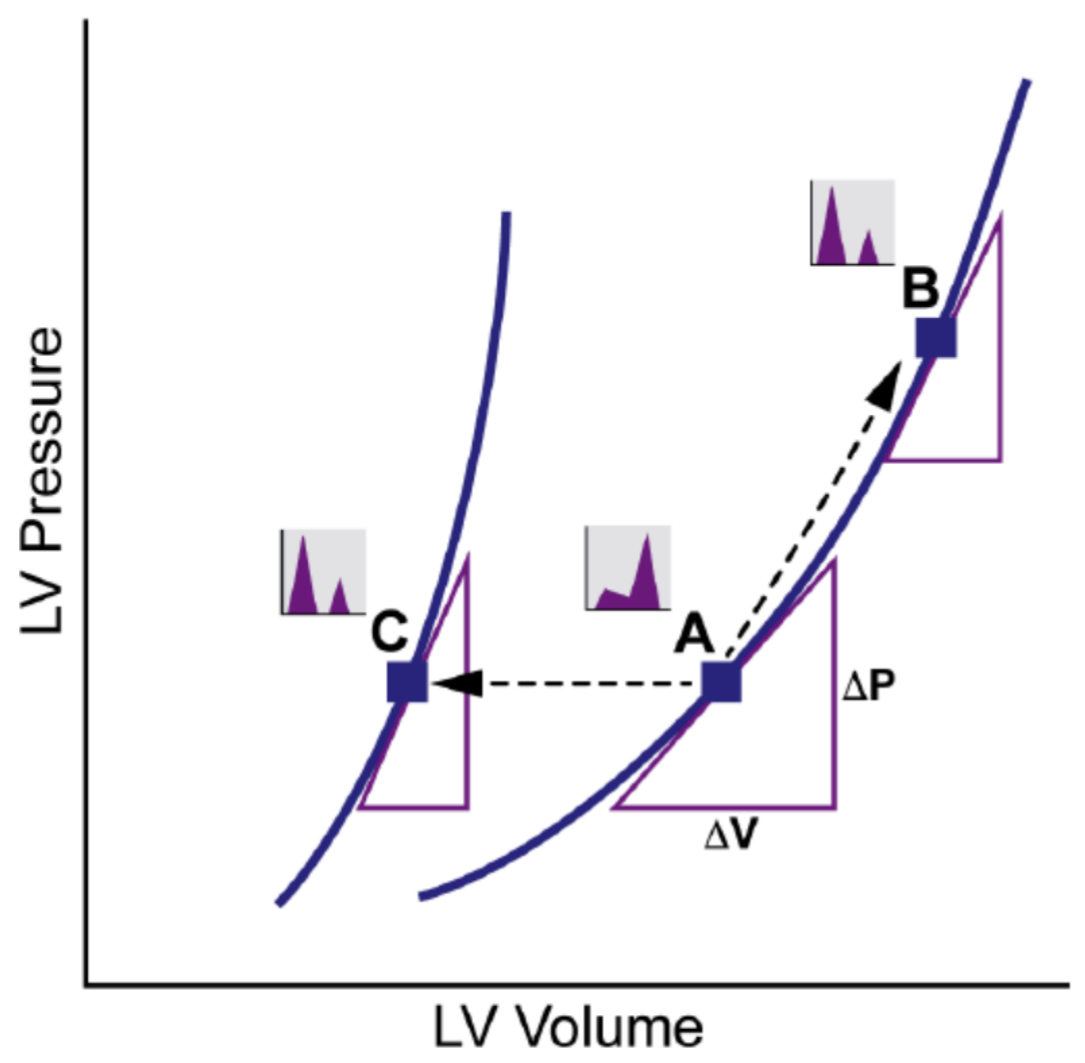

